PROCEEDINGS OF THE

AMERICAN MATHEMATICAL SOCIETY

Volume 131, Number 8, Pages 2435-2441

S 0002-9939(02)06762-X

Article electronically published on November 13, 2002

\title{
ON THE EXISTENCE OF CHAOTIC AND HYPERCYCLIC SEMIGROUPS ON BANACH SPACES
}

\author{
TERESA BERMÚDEZ, ANTONIO BONILLA, AND ANTONIO MARTINÓN
}

(Communicated by Joseph A. Ball)

\begin{abstract}
We prove that every separable infinite dimensional complex Banach space admits a hypercyclic uniformly continuous semigroup. We also prove that there exist Banach spaces admitting no chaotic strongly continuous semigroups.
\end{abstract}

\section{INTRODUCTION}

Throughout the paper, we work with complex Banach spaces, although some results are also valid in real Banach space.

A bounded linear operator $T$ on a Banach space $X$ is hypercyclic provided that there exists $x \in X$ such that $\left\{T^{n} x\right\}_{n \in \mathbb{N}}$ is dense in $X$. T is chaotic provided that it is hypercyclic and the set of periodic points is dense in $X$. A point $x$ is called periodic for $T$ if there exists some $n>1$ such that $T^{n} x=x$. This definition of chaos is consistent with the definition given by Devaney [9, page 50] for an arbitrary continuous mapping $f$ on a metric space, which requires that $f$ be transitive, the set of periodic points of $f$ be dense, and $f$ have sensitive dependence on initial conditions. The two first conditions imply the third [3]. On the other hand, Godefroy and Shapiro [12] showed that transitive operators on Fréchet spaces have sensitive dependence on initial conditions.

Interest in hypercyclic operators arises from the invariant subset problem. In fact, it is easy to check that an operator $T$ has no non-trivial invariant closed subset if and only if each non-zero vector is hypercyclic for $T$.

For more details about hypercyclicity see the excellent survey by Grosse-Erdmann 14.

In 1969, Rolewicz 23 gave the first example of a hypercyclic operator on a Banach space. He showed that if $B$ is the backward shift on $l^{2}(\mathbb{N})$, then $\lambda B$ is hypercyclic if and only if $|\lambda|>1$. Also, he wondered if for every separable infinite

Received by the editors December 8, 2001 and, in revised form, March 11, 2002.

2000 Mathematics Subject Classification. Primary 47A16, 47D03.

Key words and phrases. Chaotic semigroup, hypercyclic semigroup, hereditarily indecomposable space.

The first author was supported in part by Consejería de Educación del Gobierno de Canarias PI 2001/039 (Spain) and Universidad de La Laguna, ref:1802010204.

The second author was supported in part by DGESIC Grant PB 98-0444 (Spain) and by Consejería de Educación del Gobierno de Canarias PI 1999/105 (Spain).

The third author was supported in part by Consejería de Educación del Gobierno de Canarias PI 2001/039 (Spain). 
dimensional Banach space there exists a hypercyclic operator. This question was independently answered in the affirmative by Ansari [1] and Bernal [4], and by Bonet and Peris [6] for Fréchet spaces. On the other hand, Bonet, Martínez and Peris have recently proved that the dual of a reflexive separable hereditarily indecomposable complex Banach space admits no chaotic continuous linear operator [5].

A strongly continuous semigroup of bounded linear operator $\{T(t)\}_{t \geq 0}$ is hypercyclic provided that there exists $x \in X$ such that $\{T(t) x\}_{t \geq 0}$ is dense in $X$. Furthermore, it is chaotic if it is hypercyclic and the set $\{x \in X: \exists t>0, T(t) x=x\}$ is dense in $X$.

Hypercyclic and chaotic behaviour in strongly continuous semigroups can be viewed as the continuous time analog of the discrete time case mentioned above, and can occur when the infinitesimal generator is bounded or unbounded, but only in separable infinite dimensional spaces [8, pp. 794-795].

The following example gives a strongly continuous semigroup of bounded linear operators in $L^{2}([0, \infty), \mathbb{C})$ that is chaotic. The linear evolution problem is a convection-diffusion type equation of the form

$$
\left\{\begin{array}{l}
u_{t}(x, t)=a u_{x x}(x, t)+b u_{x}(x, t)+c u(x, t), \\
u(0, t)=0 \text { for } t \geq 0, \\
u(x, 0)=f(x) \text { for } x \geq 0 \text { with some } f \in L^{2}([0, \infty), \mathbb{C}) .
\end{array}\right.
$$

If $a, b, c>0$ with $c<\frac{b^{2}}{2 a}<1$, then the solution semigroup to this partial differential equation is chaotic. For details see [8, Example 4.12]. So, a natural problem is: Does every Banach space admit a chaotic strongly continuous semigroup?

Our aim in this paper is to prove that every separable infinite dimensional complex Banach space admits a hypercyclic uniformly continuous semigroup, and that there exist Banach spaces that admit no chaotic strongly continuous semigroups.

\section{ON HYPERCYCLIC SEMIGROUPS}

We consider the weighted $l^{1}$-space with weight sequence $\beta=\left(\beta_{i}\right)$ of positive numbers defined by

$$
\ell^{1}(\beta):=\left\{\left(x_{i}\right)_{i=1}^{\infty}: x_{i} \in \mathbb{C}, \sum_{i=1}^{\infty} \beta_{i}\left|x_{i}\right|<\infty\right\}
$$

equipped with the norm

$$
\left\|\left(x_{i}\right)\right\|:=\sum_{i=1}^{\infty} \beta_{i}\left|x_{i}\right| .
$$

If $\sup _{i \in \mathbb{N}} \frac{\beta_{i}}{\beta_{i+1}} \leq M$ for some constant $M$, then the backward shift $B$ defined by

$$
B\left(x_{1}, x_{2}, \cdots\right):=\left(x_{2}, x_{3}, \cdots\right)
$$

is a continuous linear operator on $\ell^{1}(\beta)$.

Lemma 2.1. Let $\beta=\left(\beta_{i}\right)$ be a sequence of positive numbers. If $\sup _{i \in \mathbb{N}} \frac{\beta_{i}}{\beta_{i+1}} \leq M$ for some constant $M$, then the operator $e^{B}$ is hypercyclic in $\ell^{1}(\beta)$. 
Proof. In [8, Theorem 5.2] it is proved that $e^{t B}$ is a hypercyclic semigroup. Moreover, it is derived from the proof that $e^{B}$ is a hypercyclic operator on $\ell^{1}(\beta)$, since in [8] it is proved that $T(t)$ is a hypercyclic operator for all $t$, in particular for $t=1$.

Similar results may be found in [7, Theorem 2.13].

The following two results, due to Martínez and Peris [17] and Ovsepian and Pelczynski [18, will allow us to show the existence of a hypercyclic uniformly continuous semigroup on every separable Banach space. A similar version of Lemma 2.2 for semigroups may be found in [10, Theorem 3.2].

Lemma 2.2 ([17, Lemma 2.1]). Let $T_{i}: X_{i} \rightarrow X_{i}$ be operators on separable Banach spaces $X_{i}, i=1,2$, and let $\Phi: X_{1} \rightarrow X_{2}$ be a continuous mapping with dense range such that $T_{2} \Phi=\Phi T_{1}$. If $T_{1}$ is hypercyclic (chaotic), then $T_{2}$ is also hypercyclic (chaotic).

Lemma 2.3 ([18]). Let $X$ be a separable infinite dimensional Banach space. Then there exist $\left(x_{n}\right)_{n \in \mathbb{N}} \subset X$ and $\left(f_{m}\right)_{m \in \mathbb{N}} \subset X^{*}$ satisfying the following conditions:

(1) $f_{m}\left(x_{n}\right)=\delta_{m, n}, m, n \in \mathbb{N}$.

(2) $\overline{\operatorname{span}\left\{x_{n}: n \in \mathbb{N}\right\}}=X$.

(3) $f_{m}(x)=0$, for all $m \in \mathbb{N} \Rightarrow x=0$.

(4) $\left\|x_{n}\right\|=1$, for all $n \in \mathbb{N}$ and $\sup _{m \in \mathbb{N}}\left\|f_{m}\right\|=c<\infty$.

Now, we are able to prove the main result of this section.

Theorem 2.4. Every separable infinite dimensional Banach space $X$ admits a hypercyclic uniformly continuous semigroup.

Proof. Let $\left(x_{n}\right)_{n \in \mathbb{N}} \subset X$ and $\left(f_{m}\right)_{m \in \mathbb{N}} \subset X^{*}$ as in Lemma 2.3 ,

Consider the linear continuous operator $S: X \rightarrow X$ defined by

$$
S x:=\sum_{n=1}^{\infty} \frac{1}{2^{n}} f_{n+1}(x) x_{n}
$$

given in [16, page 297].

We will prove that the operator $e^{S}$ is hypercyclic. Therefore the semigroup $e^{S t}$ is a hypercyclic uniformly continuous semigroup.

Define $\Phi: l^{1} \rightarrow X$, by $\Phi\left(\left(\alpha_{j}\right)\right):=\sum_{1}^{\infty} \alpha_{j} x_{j}$, which is a linear continuous operator with dense range.

Claim. The operator $e^{\tilde{S}}: l^{1} \rightarrow l^{1}$ is hypercyclic, where

$$
\tilde{S}\left(\left(\alpha_{j}\right)\right):=\left(\frac{\alpha_{2}}{2}, \cdots, \frac{\alpha_{n+1}}{2^{n}}, \cdots\right) .
$$

From the definition it follows that $S \Phi=\Phi \tilde{S}$ on $l^{1}$ and therefore $e^{S} \Phi=\Phi e^{\tilde{S}}$. Applying Lemma 2.2 we have that $e^{S}$ is hypercyclic in $X$.

Proof of the Claim. We define $\beta_{1}:=1$, and $\beta_{i}:=2^{1+\cdots+(i-1)}$ for $i>1$ and $\Phi_{\beta}$ : $l^{1}(\beta) \rightarrow l^{1}$, by $\Phi_{\beta}\left(\alpha_{1}, \alpha_{2}, \cdots\right):=\left(\beta_{1} \alpha_{1}, \beta_{2} \alpha_{2}, \cdots\right)$, which is a linear continuous and surjective mapping.

According to Lemma 2.1 we have that $e^{B}$ is hypercyclic on $l^{1}(\beta)$, since sup $\frac{\beta_{i}}{\beta_{i+1}} \leq$ $M$ for some constant $M$. Therefore by Lemma 2.2, the operator $e^{\tilde{S}}$ is hypercyclic because $e^{\tilde{S}} \Phi_{\beta}=\Phi_{\beta} e^{B}$. 
Remark 1. The above result implies that $e^{S}$ is hypercyclic, where $S$ is given by (2.1). In particular, this result provides a new proof of Ansari's and Bernal's result without using Salas' theorem [24] Section 3].

As a consequence of the result of Oxtoby and Ulam [19, Theorem 6] it is obtained that if $\{T(t)\}$ is a hypercyclic strongly continuous semigroup, then there exists a residual set $G \subset \mathbb{R}^{+}$, i.e. the complement of $G$ is a set of first category, such that $T(t)$ is a hypercyclic operator for all $t \in G$. This was pointed out by A. Peris to the authors in a personal communication [21] where he also proposed the following open problem.

Open Problem 1. If $\{T(t)\}$ is a hypercyclic strongly continuous semigroup, then are all $T(t)$ hypercyclic operators?

\section{ON CHAOTIC SEMIGROUPS}

Our approach to prove the existence of Banach spaces admitting no chaotic semigroups is similar to that of [5], since it is based on hereditarily indecomposable Banach spaces.

A Banach space $X$ is called hereditarily indecomposable if whenever $Y$ and $Z$ are closed infinite dimensional subspaces of $X$ satisfying $Y \cap Z=\{0\}$, then $Y+Z$ is non-closed.

Gowers and Maurey provided the first example of hereditarily indecomposable Banach space [13. In particular, they proved that every linear bounded operator $T$ on $X$ can be written as $T=\lambda I+S$, where $\lambda \in \mathbb{C}$ and $S$ is strictly singular (i.e. it has no bounded inverse on any infinite-dimensional subspace). Indeed, the spectrum of $T$ is finite or consists of $\lambda$ and a sequence $\left(\lambda_{n}\right)_{n \in \mathbb{N}}$ of eigenvalues with finite multiplicity converging to $\lambda$. Therefore, if there are not eigenvalues, the spectrum is a singleton. This fact will be used later on.

Notice that the dual of a hereditarily indecomposable space may be far from being hereditarily indecomposable [2]. However, Ferenczi proved that the space defined by Gowers and Maurey in [13], $X_{G M}$ (which is hereditarily indecomposable), satisfies that $X_{G M}^{*}$ is also hereditarily indecomposable [1] Corollary 22].

In [22, Räbiger and Ricker studied the strongly continuous semigroups on hereditarily indecomposable spaces. This reference will be an important tool for obtaining the main result of this section. In the next proposition we give some useful results of Räbiger and Ricker.

Proposition 3.1 ([22]). Let $X$ be a hereditarily indecomposable space.

(1) If $T$ is a linear operator defined in $D(T) \subset X$ with a non-empty resolvent set, then the spectrum set $\sigma(T)$ is either a finite set (possibly empty) or consists of a convergent sequence in the extended complex plane $\mathbb{C}_{\infty}:=$ $\mathbb{C} \cup\{\infty\}$ where all except at most one point in $\sigma(T)$ are eigenvalues of $T$.

(2) If $T$ is the generator of a strongly continuous group on $X$, then $T$ is a bounded linear operator.

(3) If $T$ is the generator of a strongly continuous semigroup on $X$, then the generator satisfies the spectral mapping theorem, that is,

$$
e^{t \sigma(A)}=\sigma(T(t)) \backslash\{0\}
$$

for all $t>0$. 
Recall that the spectrum of a linear operator $T$ on a normed space $X, \sigma(T)$, is defined by the complement in $\mathbb{C}$ of the resolvent set $\rho(T)$, and $\lambda \in \rho(T)$ if the operator $\lambda I-T$ has zero kernel, dense range and $(\lambda I-T)^{-1}$ is bounded.

In order to present the main result, we need an additional property.

In a similar way to [15, Proposition 2.2] we obtain the following lemma.

Lemma 3.2. Let $X$ be a Banach space and $\{T(t)\}_{t \geq 0}$ a strongly continuous semigroup such that

$$
X=M_{1} \oplus M_{2}
$$

where $M_{i}, i=1,2$, are closed subspaces with

$$
T(t) M_{i} \subset M_{i}, \quad i=1,2,
$$

for all $t \geq 0$. If $\{T(t)\}_{t \geq 0}$ is a chaotic semigroup on $X$, then its restriction on $M_{i}$, $\left\{T(t)_{M_{i}}\right\}_{t \geq 0}$ is chaotic on $M_{i}$, for $i=1,2$.

Proof. Notice that condition (3.2) is equivalent to the fact that the projection $P_{M_{i}}$, associated to the subspace $M_{i}$, commutes with $T(t)$ for all $t \geq 0$, i.e.

$$
P_{M_{i}} T(t)=T(t) P_{M_{i}}
$$

for all $t \geq 0$. Hence the proof can be readily obtained by applying a version of Lemma 2.2 for semigroups.

Remark 2. Since there is no hypercyclic strongly continuous semigroup in a finite dimensional space, the lemma implies that the decomposition given in (3.1) cannot be obtained with any finite dimensional subspace if the semigroup is hypercyclic.

Theorem 3.3. Let $X$ be a hereditarily indecomposable Banach space such that $X^{*}$ is also a hereditarily indecomposable space. Then $X$ admits no chaotic strongly continuous semigroups.

Proof. Let us suppose there exists a chaotic strongly continuous semigroup $\{T(t)\}_{t \geq 0}$, and we denote by $A$ its infinitesimal generator with domain $D(A)$. By Proposition 3.1 the strongly continuous semigroup $\{T(t)\}_{t \geq 0}$ satisfies the spectral mapping theorem, that is,

$$
e^{t \sigma(A)}=\sigma(T(t)) \backslash\{0\},
$$

for every $t>0$. Moreover, the resolvent set $\rho(A)$ cannot be an empty set, by (3.3) and since $T(t)$ is a bounded operator for all $t \geq 0$. As an application of Proposition 3.1 the spectrum of $A$ is either a finite set (possibly empty) or consists of a convergent sequence in the extended complex plane $\mathbb{C}_{\infty}$. All points, except at most one point in $\sigma(A)$, are eigenvalues of $A$.

We will proceed with the proof in four steps.

Step 1: $\sigma(A)=\{0\}$.

Since the semigroup is chaotic, there exist $x \in X \backslash\{0\}$ and $t_{0}>0$ such that $T\left(t_{0}\right) x=x$. Hence $1 \in \sigma_{p}\left(T\left(t_{0}\right)\right)$ and by the spectral mapping theorem we have that $\sigma(A) \neq \varnothing$. By [25, Theorem V.2.4] we obtain that $\sigma(A)=\sigma\left(A^{*}\right) \neq \varnothing$, and the spectrum of $A^{*}$ is a finite set or a convergent sequence in $\mathbb{C}_{\infty}$, which are eigenvalues of $A^{*}$. Notice that the limit of the sequence could be out of the point spectrum. By [8, Theorem 3.3] the point spectrum of $A^{*}$ is empty, hence $\sigma(A)=\sigma\left(A^{*}\right)=\{\lambda\}$.

Let us prove that $\lambda=0$. We have that $\{T(t)\}_{t \geq 0}$ is chaotic, hence $1 \in \sigma_{p}(T(t))$ for some $t>0$. Using the spectral mapping theorem we get that $\lambda=0$. 
Step 2: $\sigma(T(t))=\{1\}$ for all $t>0$.

Suppose that there exists $t_{0}>0$ such that $\sigma\left(T\left(t_{0}\right)\right)=\{0,1\}$. We denote by $P_{0}$ and $P_{1}$ the projections associated to the spectral sets $\sigma_{0}=\{0\}$ and $\sigma_{1}=\{1\}$, respectively, and $X_{i}=R\left(P_{i}\right)$ is the range of $P_{i}$ for $i=0,1$. Applying [25, Theorem V.9.1] we have the decomposition $X=X_{0} \oplus X_{1}$ with $\sigma\left(\left.T\left(t_{0}\right)\right|_{X_{0}}\right)=\{0\}$ and $\sigma\left(\left.T\left(t_{0}\right)\right|_{X_{1}}\right)=\{1\}$. By properties of the holomorphic functional calculus, the projections $P_{i}$ commute with every operator that commutes with $T\left(t_{0}\right)$, in particular with $T(t)$ for every $t>0$. From the definition of hereditarily indecomposable spaces it follows that at least one of $X_{i}$ must be finite dimensional. On the other hand, by Lemma 3.2 and Remark 2 we have that the dimensions of $X_{i}$ must be infinite. So we conclude a contradiction. Hence $\sigma(T(t))=\{1\}$ for all $t>0$.

Step 3: $\{T(t)\}_{t \geq 0}$ is a uniformly continuous semigroup.

By [20, Theorem I.6.5] the semigroup $\{T(t)\}_{t \geq 0}$ can be extended to a strongly continuous group, since we have that $0 \notin \sigma(T(t))=\{1\}$ for all $t>0$. According to Proposition 3.1 the infinitesimal generator is bounded and therefore the semigroup is uniformly continuous (see [20, Theorem I.1.2]).

Step 4: $X$ admits no chaotic uniformly continuous semigroup.

Suppose that there exists a chaotic uniformly continuous semigroup $\{T(t)\}_{t \geq 0}$ on $X$. By [20, Theorem I.1.2] the infinitesimal generator $A$ of $\{T(t)\}_{t>0}$ is a bounded operator on $X, T(t)=e^{t A}$, and by Step 1 we have that $\sigma(A)=\{0\}$.

Given a periodic point $x$ of $\{T(t)\}_{t \geq 0}$, there exists $t_{0}>0$ such that

$$
0=\left(T\left(t_{0}\right)-I\right) x=\left(e^{t_{0} A}-I\right) x=t_{0} A\left(\sum_{n=1}^{\infty} \frac{\left(t_{0} A\right)^{n-1} x}{n !}\right) .
$$

Define

$$
g(z):=\sum_{n=1}^{\infty} \frac{\left(t_{0} z\right)^{n-1}}{n !}
$$

which is holomorphic on $\mathbb{C}$ and $g(0)=1$. Hence by using the holomorphic functional calculus, there exists the inverse of $g(A)$ in $L(X)$, since $\sigma(A)=\{0\}$ and $g(0) \neq 0$. Therefore $0=t_{0} A g(A) x=g(A) t_{0} A x$, and consequently $A x=0$. Thus $A x=0$ for every $x$ in the dense set of periodic points of $\{T(t)\}_{t \geq 0}$. Hence $A=0$. The contradiction follows because $T(t)=I$ is not chaotic.

Remark 3. The same proof, of Theorem 3.3, works for $X$ to be a dual of a reflexive separable hereditarily indecomposable complex Banach space of Gowers and Maurey, using [20, Theorem II.2.4] instead of the spectral mapping theorem.

To finish, we want to pose the following problem.

Open Problem 2. Characterize those Banach spaces $X$ which admit a chaotic strongly continuous semigroup.

\section{ACKNOWLEDGMENTS}

We thank Alfredo Peris and Hassan Emamirad who made some interesting comments about the paper. We also thank the referee for many helpful suggestions.

\section{REFERENCES}

1. S. I. Ansari, Existence of hypercyclic operators on topological vector spaces, J. Funct. Anal. 148 (1997), 384-390. MR 98h:47028a 
2. S. A. Argyros, V. Felouzis, Interpolating hereditarily indecomposable Banach spaces, J. Amer. Math. Soc. 13 (2000), 243-294. MR 2002b:46021

3. J. Banks, J. Brooks, G. Cairns, G. Davis, P. Stacey, On Devaney's definition of chaos, Amer. Math. Monthly 99 (1992), 332-334. MR 93d:54039

4. L. Bernal-González, On hypercyclic operators on Banach spaces, Proc. Amer. Math. Soc. 127 (1999), 1003-1010. MR 99f:47010

5. J. Bonet, F. Martínez-Giménez, A. Peris, A Banach space which admits no chaotic operator, Bull. London Math. Soc. 33 (2001), 196-198. MR 2001m:47015

6. J. Bonet and A. Peris, Hypercyclic operators on non-normable Fréchet spaces, J. Funct. Anal. 159 (1998), 587-595. MR 99k:47044

7. R. DeLaubenfels, H. Emamirad, Chaos for functions of discrete and continuous weighted shift operators, Ergodic Theory Dynam. Systems 21 (2001), 1411-1427.

8. W. Desch, W. Schappacher and G. F. Webb, Hypercyclic and chaotic semigroups of linear operators, Ergodic Theory Dynam. Systems 17 (1997), 793-819. MR 98j:47083

9. R. L. Devaney, "An introduction to chaotic dynamical systems", Addison Wesley, Reading, MA, 1989. MR 91a:58114

10. H. Emamirad, Hypercyclicity in the scattering theory for linear transport equation, Trans. Amer. Math. Soc. 350 (1998), 3707-3716. MR 98k:47077

11. V. Ferenczi, Quotient hereditarily indecomposable Banach spaces, Canad. J. Math. 51 (1999), 566-584. MR 2000k:46012

12. G. Godefroy, J. H. Shapiro, Operators with dense, invariant, cyclic vector manifolds, J. Funct. Anal. 98 (1991), 229-269. MR 92d:47029

13. W. T. Gowers, B. Maurey, The unconditional basic sequence problem, J. Amer. Math. Soc. 6 (1993), 851-874. MR 94k:46021

14. K.-G. Grosse-Erdmann, Universal families and hypercyclic operators, Bull. Amer. Math. Soc. 36 (1999), 345-381. MR 2000c:47001

15. D. A. Herrero, Limits of hypercyclic and supercyclic operators, J. Funct. Anal. 99 (1991), 179-190. MR 92g:47026

16. G. Herzog, On linear operators having supercyclic operators, Studia Math. 103 (1992), 295298. MR 93k:47033

17. F. Martínez-Giménez, A. Peris, Chaos for backward shift operators, Int. J. of Bifurcation and Chaos 12 (2002), 1703-1715.

18. R. I. Ovsepian, A. Pelczynski, On the existence of a fundamental total and bounded biorthogonal sequence in every separable Banach space, and related constructions of uniformly bounded orthonormal systems in $L^{2}$, Studia Math. 54 (1975), 149-159. MR 52:14942

19. J. C. Oxtoby, S. M. Ulam, Measure-preserving homeomorphisms and metrical transitivity, Ann. Math. 42 (1941), 874-920. MR 3:211b

20. A. Pazy, "Semigroups of linear operators and applications to partial differential equations" (Applied Mathematical Sciences) 44 Springer-Verlag, New York, 1983. MR 85g:47061

21. A. Peris, Personal communication.

22. F. Räbiger, W. J. Ricker, $C_{0}$-groups and $C_{0}$-semigroups of linear operators on hereditarily indecomposable Banach space, Arch. Math. 66 (1996), 60-70. MR 96i:47070

23. S. Rolewicz, On orbits of elements, Studia Math. 32 (1969), 17-22. MR 39:3292

24. H. N. Salas, Hypercyclic weighted shifts, Trans. Amer. Math. Soc. 347 (1995), 993-1003. MR 95e: 47042

25. A. C. Taylor, D. C. Lay, "Introduction to Functional Analysis" (2nd edition), John Wiley \& Sons, New York, 1980. MR 81b:46001

Departamento de Análisis Matemático, Universidad de La Laguna, 38271 La Laguna

(Tenerife), Spain

E-mail address: tbermude@ull.es

Departamento de Análisis Matemático, Universidad de La Laguna, 38271 La Laguna (TEnerife), Spain

E-mail address: abonilla@ull.es

Departamento de Análisis Matemático, Universidad de La Laguna, 38271 La Laguna (TENERIFE), SPAIN

E-mail address: anmarce@ull.es 